\title{
Pressure Sensor Interface Circuit Based on Silicon Carbide Electronics for Harsh Environment Operation
}

\author{
Joseph Samy Albert Riad \\ ECE Department \\ Faculty of Engineering, Ain Shams \\ University \\ Cairo, Egypt \\ joseph2@eng.asu.edu.eg
}

\author{
Mourad N. El-Gamal \\ ECE Department \\ McGill University \\ Montréal, Canada
}

\author{
Hani Fikry Ragai \\ ECE Department \\ Faculty of Engineering, Ain Shams \\ University \\ Cairo, Egypt
}

\begin{abstract}
Pressure sensing in harsh environments poses many difficulties. The major challenge is implementing a sensor interface circuit capable of operation at high temperatures $\left(z 300^{\circ} \mathrm{C}\right.$ ) for extended periods of time. Conventional, siliconbased electronics are not suitable for operation in such conditions due to their sizable leakage currents and junction instability. In this work, a wireless solution based on silicon carbide electronics is presented. The proposed solution follows a simple design procedure and exhibits robustness to the large process variations typically associated with silicon carbide technology.
\end{abstract}

Keywords—silicon carbide; harsh environment;sensor interface

\section{INTRODUCTION}

Among the many applications for MEMS pressure sensors, harsh environment applications have garnered a lot of recent interest [1-3]. An environment is considered harsh if it subjects a device to high pressures and temperatures, chemically corrosive medium or high levels of radiation. Such environments arise typically in automotive, aerospace, industrial and military applications. Typical applications include monitoring of internal combustion engines, aircraft wing flaps and industrial process control.

One of the major challenges facing sensor design for harsh environment applications is the design of an interface circuit capable of high temperature $\left(300^{\circ} \mathrm{C}\right)$ for extended periods of time. Electronic circuits operating at elevated temperatures face many issues that limit the maximum temperature at which they can function correctly; namely, leakage current, junction stability and intrinsic conduction effects [4]. For these reasons, silicon-based electronics are inappropriate for the design of the sensor interface and a new material is required. Silicon carbide ( $\mathrm{SiC})$ has proven to be a suitable material for this purpose due to its large bandgap energy and extremely low intrinsic carrier concentration [4].

In this work, the process of designing an interface circuit based on $\mathrm{SiC}$ electronics is detailed along with simulation results demonstrating the correct functionality and robustness of the circuit in question. The paper starts by describing the major design issues involved and motivates the choice of topology; this is followed by detailed analysis of the chosen topology and a description of the design flow utilized in order to meet the design constraints. Simulation results and discussion follow, demonstrating the correct functioning of the circuit. Finally, the major conclusions and suggestions for future work conclude the paper.

\section{DESIGN IsSUES AND TOPOLOGY CHOICE}

The issues involved in the design of the sensor interface circuit are mainly due to the shortcomings of the SiC fabrication process and the constraints imposed by the harsh operating conditions of high temperature as described before.

The main challenge in the manufacturing of $\mathrm{SiC}$, is that, owing to its poor interface with silicon dioxide, surface mobility in $\mathrm{SiC}$ devices to date remains nearly an order of magnitude lower than that of silicon [5]. The fabrication technology, still considered "nascent" [5], lacks reproducibility [6]. This low reproducibility renders circuit design especially challenging and requires simple circuit topologies that are able to tolerate large variation in performance parameters.

The fact that the sensor and its interface circuit must be present in a harsh environment that is unsafe for humans requires a connection between the measurement environment and the sensor's human users. Opting for a cable connection leads to a bulky system that is difficult to maintain and challenging to design due to the parasitics of the connecting leads. For this reason, a wireless solution is desirable for transmitting the measured signal outside the harsh measurement environment without the need for connecting cables.

Even though SiC-based operational amplifiers and other circuits have already been demonstrated to function well over a wide temperature range [6-8], circuit architectures are usually complicated and involve adaptive biasing schemes in order to accommodate the large process variations and the wide temperature range. Furthermore, conventional capacitive sensor interface circuits, usually based on switched capacitor amplifiers, will either require cable connection or an additional wireless transmitter circuit for the transmission of the measurement data outside the measuring environment, which further adds to the complexity of the system.

For these reasons, the single-transistor Colpitts oscillator circuit architecture used in [1] was preferred. The simple de- 
sign is attractive due to its robustness to variation, and offers a means for safely transmitting the measured signal outside the harsh environment without the need for connecting cables. This is accomplished via inductive coupling with the inductor coil of the oscillator circuit as successfully demonstrated in [1] and [3]. The circuit architecture is shown in fig. 1. In the figure, the variable capacitor $(\boldsymbol{C})$ is meant to represent the pressure sensor.

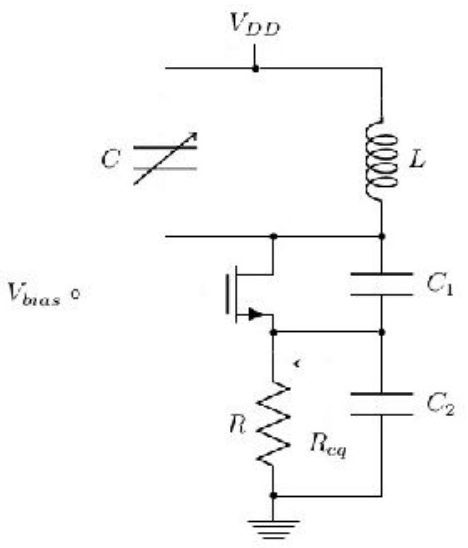

Fig. 1. Colpitts oscillator circuit selected for the sensor interface.

\section{CIRUICT ANALYSIS AND DESIGN}

As explained above, $\mathrm{SiC}$ process technology is still considered immature. This means that no standard circuit models for SiC MOSFETs currently exist; therefore, in order to enable meaningful circuit design, a simplified SPICE model was extracted based on published results [9] and used in this work.

\section{A. Circuit Analysis}

The analysis of the Colpitts oscillator circuit presented here follows closely the analysis in [10], while adapting it to account for the sensor capacitance. Fig. 2 shows the circuit's small signal model, where $R_{\oplus q}$ is the resistance seen looking into the transistor's source as shown in fig. 1 and $R_{L}$ represents the losses in the inductor coil. As can be noted from the figure, the capacitive feedback network forms, along with $R_{e q}$, a high pass filter whose frequency response is shown in fig. 3 and whose cutoff frequency and phase response are given by [10]:

$$
\begin{gathered}
\omega_{c}=\frac{1}{R_{a q}\left(C_{1}+C_{2}\right)} \\
\phi(\omega)=\frac{\pi}{2}-\arctan \left(\frac{\omega}{\omega_{c}}\right)
\end{gathered}
$$
$\frac{\omega}{\omega_{c}}$

and thus, for correct operation, a large value for the ratio

$\omega_{c}$ is required.

Analyzing the small signal model yields the following equations:

$$
\left[\begin{array}{cc}
\frac{1}{R_{L}}+\frac{1}{s L}+s\left(C+C_{1}\right) & -\left(s C_{1}+g_{m}\right) \\
-s C_{1} & s\left(C_{1}+C_{2}\right)+\frac{1}{R_{s q}}
\end{array}\right]\left[\begin{array}{l}
v_{d} \\
v_{s}
\end{array}\right]=0
$$

For the circuit to have a non-trivial solution the determinant of the coefficients matrix must vanish and having sustained oscillations requires the circuit's poles to be on the $j \omega$ axis in the $s$ plane. With these conditions, the circuit's oscillating frequency and condition of oscillation may be found as follows:

$$
\begin{gathered}
\omega_{o s c}=\omega_{0} \sqrt{1+\frac{1}{Q \frac{\omega}{\omega_{c}}}} \\
g_{m}>g_{m}^{*}=\frac{\omega_{o s c}\left(C_{1}+C_{2}\right)}{Q\left(1-\omega_{o s c}^{2} L C\right)}
\end{gathered}
$$

where $Q$ is the inductor's unloaded quality factor and

$$
\omega_{0}=\frac{1}{\sqrt{L\left(C+\frac{C_{1} C_{2}}{C_{1}+C_{2}}\right)}}
$$

is the tank circuit's resonant frequency.

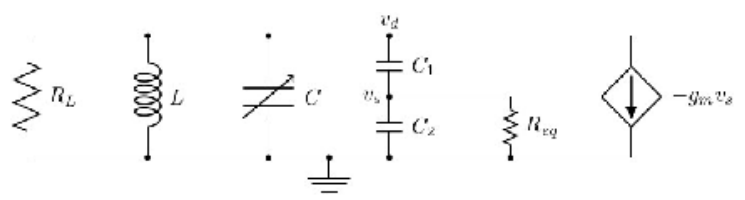

Fig. 2. Colpitts oscillator circuit small signal model.
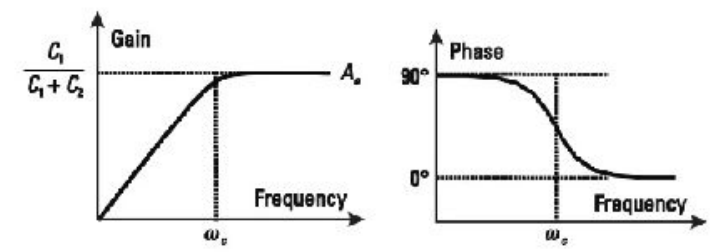

Fig. 3. Frequency response of highpass filter formed by the capacitive feedback network of the Colpitts oscillator. Courtesy of [10]

On the other hand, the feedback network's cutoff frequency is given by

$$
\omega_{c} \cong \frac{g_{m}}{C_{1}+C_{2}}
$$

This sets an upper limit on device $g_{m}$ in order to ensure that $\omega_{o s c}$ is sufficiently larger than $\omega_{c}$ for correct operation $\left(\phi\left(\omega_{o s c}\right) \cong \mathbf{0}\right)$. It is thus found that $g_{m}$ is constrained between two limits:

$$
\frac{\omega_{0}\left[C+C_{1}(1-n)\right]}{Q n(1-n)}<g_{m}<\frac{\omega_{0} C_{1}}{n}
$$


where $n=\frac{C_{2}}{C_{1}+C_{2}}$ venience. This sets an upper limit on sensor capacitance that may be deduced in terms of two new factors $M$ and $N$ introduced

$$
\begin{aligned}
& M=\frac{g_{m}}{g_{m}^{*}} \\
& N=\frac{W_{o s c}}{\omega_{c}}
\end{aligned}
$$

The upper limit on sensor capacitance may thus be expressed as

$$
C_{\max }=C_{1}(1-n)\left(\frac{Q}{M N}-1\right)
$$

This demonstrates that the tank circuit quality factor severely limits the maximum allowed sensor capacitance and hence sensitivity. Since integrated inductors usually have low quality factors, and due to the lack of models for integrated inductors over a SiC substrate, an off-chip inductor coil is chosen for the current implementation.

\section{B. Design Procedure}

Since wireless transmission is targeted, an operating frequency in the ISM band is chosen for the oscillator. The required operating pressure range and allowed ISM bandwidth limit the overall system sensitivity (in $\mathrm{Hz} / \mathrm{Pa}$ ). The system sensitivity $S$ may be thought of as the product of the sensor sensitivity $S_{g}$ and the oscillator sensitivity $S_{a}$. It can be shown that $S_{s}$ is directly proportional to sensor capacitance and $S_{o}=-\frac{f_{o s c}}{C_{\theta q}}$ where $C_{\nexists q}=C+n C_{1}$. From these equations, it may be shown that $S$ increases as $n$ decreases. For this reason, $n$ is fixed at 0.5 in order to strike a compromise between system sensitivity and simplicity of matching the feedback capacitors.

The operating frequency is chosen to be $433 \mathrm{MHz}$, in the mid range of the ISM band, to ensure that both inductor and transistor will be of reasonable size. The inductor coil chosen for the circuit is of nominal inductance $3.85 \mathrm{nH}$ and has an unloaded quality factor of 100 based on typical commercial device parameters [11].

With these facts in mind, the following design procedure was used to complete the oscillator design:

1. From the chosen operating frequency and coil inductance, the equivalent tank circuit capacitance $\left(C_{\theta q}\right)$ is obtained.

2. Oscillator sensitivity $\left(S_{0}\right)$ is calculated from $C_{\theta q}$.

3. Given the operating pressure range and allowed ISM channel bandwidth, the overall system sensitivity is fixed and hence, $S_{s}$ may be calculated from $S_{0}$.
4. Using the sensor sensitivity, the pressure sensor is designed according to the method described in [12] and its capacitance in the middle of the pressure range $(C)$ is extracted. Since $C q$ is known and $\mathrm{n}=0.5$, the values of both $C_{1}$ and $C_{2}$ may now be obtained.

5. Using the upper limit on sensor capacitance derived above, the value of the product $M N$ may now be found. As a simple heuristic, the design targets to place the typical value of $g_{m}$ "midway" between both extremes to allow for maximum variation in both directions. Thus both $M$ and $N$ are fixed at their geometrical mean.

6. The supply voltage should be set to a large enough value to ensure that the transistor operates in saturation in the typical case. The source resistance

$(\boldsymbol{R})$ should also be quite large compared to $\overline{g_{m}}$ in order to ensure that the foregoing analysis (in particular, the approximate value of $\omega_{e}$ used) is valid. For simplicity, $R$ is set to $\frac{\mathbf{5}}{g_{m}}$. With everything else fixed, the transistor may now be sized by fixing $L$ at $1 \mathrm{~m}$ (a typical value for current SiC devices) and finding $W$, thereby completing the design.

The above procedure was used to design the interface circuit for a sensor operating in the pressure range 100-300 atm. and the resulting design is given in table $\mathrm{I}$.

TABLE I.

PARAMETERS OF THE DESIGNED SENSOR INTERFACE CIRCUIT

\begin{tabular}{|l|c|}
\hline Parameter & Value \\
\hline$C_{\mathbf{1}}$ & $69 \mathrm{pF}$ \\
\hline$C_{\mathbf{Z}}$ & $69 \mathrm{pF}$ \\
\hline $\boldsymbol{R}$ & 133 \\
\hline$L$ & $3.85 \mathrm{nH}$ \\
\hline$W$ & $58600 \mathrm{~m} / 1 \mathrm{~m}$ \\
\hline$L$ & \\
\hline$I_{D S}$ & $24 \mathrm{~mA}$ \\
\hline
\end{tabular}

\section{SimUlation RESUlTS AND DisCUSSION}

\section{A. Typical Case}

The foregoing design was simulated and the simulation results are presented in this section. Fig. 3 shows the frequency response of the oscillator exhibiting zero phase response at the desired center frequency with adequate gain. Fig. 4 shows the transient simulation results demonstrating the correct build up and sustainment of oscillations. The circuit thus demonstrates adequate functionality under typical conditions. 


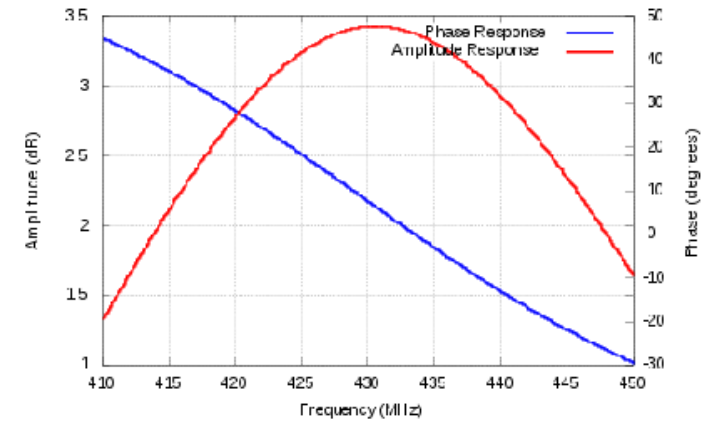

Fig. 4. Frequency response of the designed oscillator circuit.

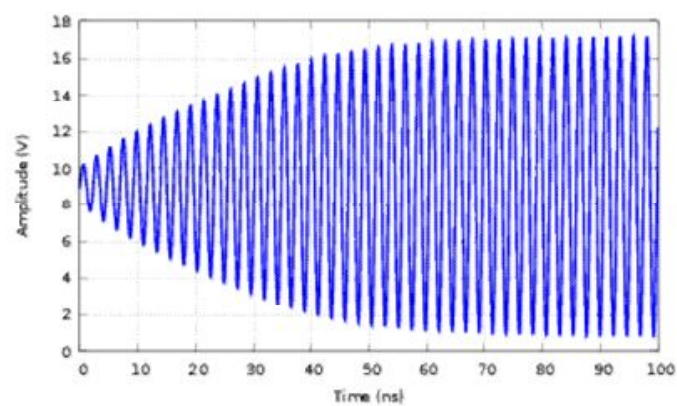

Fig. 5. Oscillation startup and sustainment exhibited by the designed oscillator circuit.

\section{B. Verification Across Process, Voltage and Temperature Variations}

In order to test the robustness of the design to variations in process parameters, supply voltage and temperature, a simple simulation script was devised to test the circuit (pressure sensor and oscillator) at all the combinations of the corner cases shown in table II. The variation in threshold voltage is taken as an example of process variations and is calculated based on $\pm 25 \%$ substrate doping tolerance reported in [13].

TABLE II. CORNER CASES USED TO VERIFY THE OSCILLATOR DESIGN

\begin{tabular}{|c|c|c|}
\hline Parameter & Minimum & Maximum \\
\hline$V_{\boldsymbol{D D}}(\mathrm{V})$ & 9 & 11 \\
\hline$\frac{\Delta V_{\text {th }}}{V_{\text {th }}}$ & -0.125 & +0.125 \\
\hline Temperature $\left({ }^{\circ} \mathrm{C}\right)$ & 27 & 300 \\
\hline
\end{tabular}

With the pressure sensor structure being modeled via Verilog-A, the pressure parameter was swept and a plot of the oscillator's output frequency as a function of pressure was constructed for each corner case. The results of this analysis are shown in figure 6 . It is seen that PVT variations cause a maximum frequency deviation of about $0.45 \mathrm{MHz}$. If we assume that ambient temperature will remain high in the measurement environment and omit temperature variations, we see that process and voltage variations cause a maximum frequency deviation of about $0.35 \mathrm{MHz}$ and this sets a limit on the minimum resolution achievable by the system. It should be noted that the ISM bandwidth for the $433 \mathrm{MHz}$ frequency is $1.74 \mathrm{MHz}$, which shows that this resolution is quite coarse due to the large variability in process parameters.

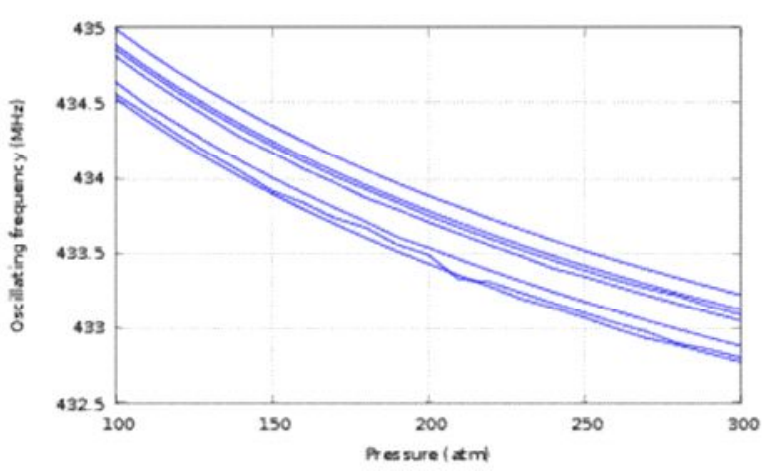

Fig. 6. Tuning (frequency vs. pressure) of the interface circuit across PVT.

\section{CONCLUSIONS AND SUGGESTIONS FOR FUTURE WORK}

This work has presented detailed design and simulation for a Colpitts oscillator circuit meant as a capacitive sensor interface circuit operating at high temperature. It has been demonstrated that the circuit functions correctly in the typical case and is robust to PVT variations.

It was further concluded that process variability remains a severe limiting factor on circuit and system performance because it limits the system resolution to a very coarse value (roughly $20 \%$ of the allowed bandwidth).

As avenues of future work, it is suggested to first mitigate the severe variations in oscillation frequency using circuit techniques and then to implement a power supply circuit (to supply $V_{D D}$ ) that is capable of operation in harsh environments.

\section{REFERENCES}

[1] Run Wang, Wen H. Ko, and Darrin J. Young. "Silicon-CarbideMESFET-Based $400^{\circ} \mathrm{C}$ MEMS Sensing and Data Telemetry." IEEE Sensors Journal, 5:1389-1394, 2005.

[2] Y. Hezarjaribi et al. "Capacitive Pressure Sensors Based on MEMS, Operating in Harsh Environments", ICSE 2008 Proceedings

[3] Jie Yang. "A harsh environment wireless pressure sensing solution utilizing high temperature electronics." Sensors, 13(3):2719-2734, 2013.

[4] Wai-Kai Chen. The VLSI Handbook, Second Edition (Electrical Engineering Handbook). CRC Press, Inc., Boca Raton, FL, USA, 2006.

[5] Stephen Daley Arthur, Dale Marius Brown, and Kevin Sean Matocha. Silicon carbide semiconductor structures, devices, and methods for making the same, 022011.

[6] A.C. Patil. Silicon Carbide JFET Integrated Circuit Technology for High-temperature Sensors. Ph.d. thesis, Case Western Reserve University, 2009.

[7] J.-S. Chen, and K. Kornegay, "Design of a Process Variation Tolerant CMOS Opamp in 6H-SiC Technology for High-Temperature Operation" "Circuits and Systems I: Fundamental Theory and Applications, IEEE Transactions on, 45(11):1159-1171., 1998

[8] A. Maralani, and M. Mazzola, "The Design of an Operational Amplifier Using Silicon Carbide JFETs". Circuits and Systems I: Regular Papers, IEEE Transactions on, 59(2):255 -265., 2012

[9] Cheng-Po Chen Avinash S. Kashyap and Vinayak Tilak. "Compact Modeling of Silicon Carbide Lateral MOSFETS for Extreme Environment Integrated Circuits". Technical report, General Electric Global Research Center, 2011.

[10] Calvin Plett John Rogers. Radio Frequency Integrated Circuit Design. Artech House, 2003.

[11] http://www.coilcraft.com/micro.cfm 
[12] J. Riad, M. El-Gamal, and H. Ragai, "Analytical Speedup for the Optimal Design of MEMS Touch Mode Capacitive Pressure Sensors", International Journal of Enhanced Research in Science, Technology and Engineering, Vol. 3, Issue 1, 2014
[13] Cree Inc., "Cree silicon carbide substrates and epitaxy". http://www.cree.com/, 2013 